\title{
An Optimal Link Layer Model for Multi-Hop MIMO Networks
}

\author{
Yi Shi \\ Jia Liu \\ Canming Jiang \\ Cunhao Gao \\ Y. Thomas Hou \\ The Bradley Department of Electrical and Computer Engineering \\ Virginia Polytechnic Institute and State University, Blacksburg, VA 24061, USA
}

\begin{abstract}
The rapid advances of MIMO to date have mainly stayed at the physical layer. Such fruits have not been fully benefited at the network layer mainly due to the computational complexity associated with the matrix-based model that MIMO involves. Recently, there are some efforts to simplify link layer model for MIMO so as to ease research for the upper layers. These models only require numeric computations on MIMO's degrees-of-freedom (DoFs) for spatial multiplexing (SM) and interference cancellation (IC) to obtain a feasible rate region. Thus, these models are much simpler than the original matrixbased model from the communications world. However, none of these DoF-based models is shown to achieve the same rate region as that by the matrix-based model. In this paper, we re-visit this important problem of MIMO modeling. Based on accurate accounting of how DoFs are consumed, we develop a simple link layer model for multi-hop MIMO networks. We show that this model is optimal in the sense of achieving the same rate region as that by the matrix-based model under SM and IC for any network topology. This work offers an important building block for theoretical research on multi-hop MIMO networks.
\end{abstract}

\section{INTRODUCTION}

MIMO is a powerful physical layer technology to increase link capacity [2], [22]. However, most of the technological advances of MIMO to date have stayed at the physical layer [2], [8], [10], [11], [22]. Fundamental understanding and optimal results on translating MIMO capability to upper layers remain very limited. The major technical barrier in this stagnation is the lack of a simple and accurate MIMO model that is amenable for cross-layer optimization. Existing models for MIMO based on physical layer channel gain matrices, although accurate, are cumbersome to handle, due to the computational complexity associated with matrix manipulations. As a result, networking research based on these models has resulted in very limited success [5], [12].

Recognizing the difficulties in dealing with MIMO channel gain matrices, some researchers have attempted to simplify MIMO models for networking research (see [1], [7], [13], [17], [21]). These models were built upon degrees of freedom (DoFs) representation [22, Chapter 7] for spatial multiplexing (SM) and interference cancellation (IC) [4], [14], [20]. Under this approach, a node can exploit its DoFs for either SM or IC such that higher data rate can be achieved. Instead of carrying complex manipulations on matrices, DoF-based MIMO models only require some numeric computations to identify a feasible rate region. Such models have since been applied to solve throughput optimization problems [1], [7], [13] and to design MAC protocols [17], [21]. A significant advantage of DoF-based models is that problem formulations under this approach share similar structure as those for singleantenna wireless networks. Thus, one can develop solutions to multi-hop MIMO networks by exploiting the vast experience and literature that has been accumulated for single-antenna multi-hop wireless networks.

\section{A. Limitation of Existing DoF-based Models}

Although DoF-based modeling offers significant advantages over traditional matrix-based representation, none of existing DoF-based models is shown to be optimal. By optimality, we mean that a simple DoF-based model can achieve the same rate region as that by the original matrix-based model under SM and IC. Existing DoF-based models [1], [7], [13], [17], [21] focused on identifying sufficient conditions for feasible data streams under SM and IC. In particular, Bhatia and Li [1] found that IC could be done by both transmitters and receivers. In [17], Park et al. found that to have a newly active transmission join other ongoing transmissions, one could let the newly active transmitter and receiver to cancel interference. Sundaresan et al. [21] found that IC could be done by receivers only. However, Hamdaoui and Shin [7] found that for each interference between two links, one could let either a transmitter or a receiver to cancel this interference, but not both. Note that identifying which node should perform IC is not a trivial problem. This problem is important as an incorrect choice of node for IC can yield an infeasible solution. Due to this difficulty, Hamdaoui and Shin rolled back to the approach in [1] to ensure feasibility, i.e., to have both transmitter and receiver use their DoFs for IC. Therefore, none of DoFbased models in [1], [7], [17], [21] can achieve the same rate region as that by the matrix-based model. In [13], Liu et al. proposed a node-level ordering scheme to identify which node should perform IC. In particular, a transmitter should cancel its interference to all non-intended receivers ordered before itself and a receiver should cancel interference from all non-intended transmitters ordered before itself. A model based on a nodelevel ordering can guarantee feasibility. Such a model is shown to achieve a larger feasible rate region than that by previous DoF models. However, there was no proof of optimality.

\section{B. Main Contributions}

The goal of this paper is to develop an optimal DoF-based link layer model for multi-hop MIMO networks to achieve the same rate region as that by the matrix-based model under SM and IC for any network topology. Our main contributions can be summarized as follows. 
- To avoid any loss of rate region, we start from the matrix-based model to formally derive how DoFs are consumed by SM and IC in a multi-hop MIMO network. In particular, we show that the number of DoFs consumed by IC can be determined by a vector-level ordering.

- Further, we prove that for the purpose of achieving the same rate region, it is sufficient to work with a "nodelevel" ordering instead of a vector-level ordering.

- Based on the above analysis, we propose an optimal link layer model for any MIMO network topology. Same as previous DoF-based models, our model only requires simple numeric computations to characterize a feasible rate region for a multi-hop MIMO network. But unlike previous models, our model is proved to achieve the same rate region as that by the matrix-based model.

\section{Paper Organization}

The remainder of this paper is organized as follows. In Section II, we offer necessary background on MIMO and the matrix-based model for SM and IC. Section III analyzes DoF consumption in the matrix-based model. In Section IV, we develop our new DoF-based link layer model for multi-hop MIMO networks that retains the simplicity of existing DoFbased models while achieving the same rate region as that by the matrix-based model. We also compare our DoF-based model to the matrix-based model and a previous model in terms of rate region and complexity. In Section V, we apply our model to study a cross-layer optimization problem for a multi-hop network. Section VI concludes this paper.

\section{LINK LAYER MODEL FOR MIMO NETWORKS: A PRIMER}

In this section, we formalize a matrix-based model for linear MIMO transceiver [4], [14], [20] for SM and IC and discuss its limitation. This model will also serve as a starting point in our new DoF-based modeling in Sections III and IV.

Consider a multi-hop MIMO network with $N$ nodes. Suppose that there are $L$ possible links in this network. Denote $\operatorname{Tx}(l)$ and $\operatorname{Rx}(l)$ the transmitter and receiver of link $l$, $1 \leq l \leq L$, respectively. The number of antennas at $\operatorname{Tx}(l)$ and $\operatorname{Rx}(l)$ are denoted as $A_{\mathrm{Tx}(l)}$ and $A_{\mathrm{Rx}(l)}$, respectively. Due to potential interference, these links may not be active at the same time. We consider a time slot based scheduling. That is, we consider a time frame with $T$ equal-length time slots and within a time slot $t, 1 \leq t \leq T$, only a subset of these $L$ links can be active. Since a MIMO link can support multiple data streams by SM, we denote $z_{l}[t]$ the number of data streams on link $l$ in time slot $t$. For simplicity, we assume that one data stream corresponds to one unit data rate, ${ }^{1}$ then the average rate of each link $l$ over $T$ time slots is

$$
c_{l}=\frac{1}{T} \sum_{t=1}^{T} z_{l}[t] \quad(1 \leq l \leq L) .
$$

\footnotetext{
${ }^{1}$ In general, the achievable rate by one data stream depends on the channel gain.
}

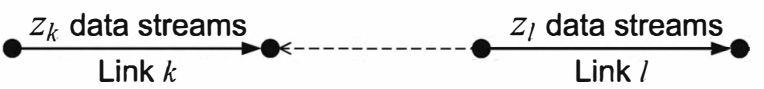

Fig. 1. Interference cancellation between two MIMO links.

We now describe SM and IC and their constraints. ${ }^{2}$

SM. Spatial multiplexing refers that a transmitter multiplexes several data streams in spatial domain when sending to its receiver. For a link $l$ in time slot $t$, denote $s_{l j}[t]$ the signal of data stream $j, 1 \leq j \leq z_{l}[t]$. To transmit $z_{l}[t]$ data streams, transmitter $\operatorname{Tx}(l)$ chooses an $A_{\operatorname{Tx}(l)} \times 1$ transmit weight vector $\mathbf{u}_{l j}[t]$ for each data stream $j$ and sends the combined signal vector $\sum_{j=1}^{z_{l}[t]} \mathbf{u}_{l j}[t] s_{l j}[t]$ through its $A_{\mathrm{Tx}(l)}$ antennas. Denote $\mathbf{H}_{(l, l)}$ the $A_{\mathrm{Tx}(l)} \times A_{\mathrm{Rx}(l)}$ channel gain matrix between nodes $\operatorname{Tx}(l)$ and $\operatorname{Rx}(l)$. The signal vector at receiver $\operatorname{Rx}(l)$ 's $A_{\mathrm{Rx}(l)}$ antennas is $\left(\sum_{j=1}^{z_{l}[t]} \mathbf{u}_{l j}[t] s_{l j}[t]\right)^{\dagger} \mathbf{H}_{(l, l)}$. Receiver $\operatorname{Rx}(l)$ uses an $A_{\mathrm{Rx}(l)} \times 1$ receive weight vector $\mathbf{v}_{l i}[t]$ to receive data stream $i, 1 \leq i \leq z_{l}[t]$. The received signal $r_{l i}[t]$ for data stream $i$ is

$$
\begin{aligned}
r_{l i}[t]= & \left(\sum_{j=1}^{z_{l}[t]} \mathbf{u}_{l j}[t] s_{l j}[t]\right)^{\dagger} \mathbf{H}_{(l, l)} \mathbf{v}_{l i}[t] \\
= & \left(\left(\mathbf{u}_{l i}[t]\right)^{\dagger} \mathbf{H}_{(l, l)} \mathbf{v}_{l i}[t]\right) \cdot s_{l i}[t] \\
& +\sum_{1 \leq j \leq z_{l}[t]}^{j \neq i}\left(\left(\mathbf{u}_{l j}[t]\right)^{\dagger} \mathbf{H}_{(l, l)} \mathbf{v}_{l i}[t]\right) \cdot s_{l j}[t] .
\end{aligned}
$$

The received signal $r_{l i}[t]$ can be the same as $s_{l i}[t]$, if we choose appropriate $\mathbf{u}$ and $\mathbf{v}$ vectors such that

$$
\begin{array}{rlc}
\left(\mathbf{u}_{l i}[t]\right)^{\dagger} \mathbf{H}_{(l, l)} \mathbf{v}_{l i}[t]=1 & \left(1 \leq i \leq z_{l}[t]\right) \\
\left(\mathbf{u}_{l i}[t]\right)^{\dagger} \mathbf{H}_{(l, l)} \mathbf{v}_{l j}[t]=0 & \left(1 \leq i, j \leq z_{l}[t], j \neq i\right) .
\end{array}
$$

IC. In addition to SM, a MIMO node can cancel interference so that several links can be active simultaneously in the same vicinity. ${ }^{3}$ This is also known as spatial reuse [7], [14]. We now consider two links $l$ and $k$ in a time slot $t$ (see Fig. 1), where the receiver on link $k$ is interfered by the transmitter on link $l$. As discussed, transmitter $\operatorname{Tx}(l)$ sends the combined signal vector $\sum_{i=1}^{z_{l}[t]} \mathbf{u}_{l i}[t] s_{l i}[t]$ through its $A_{\mathrm{Tx}(l)}$ antennas. Denote $\mathbf{H}_{(l, k)}$ the $A_{\operatorname{Tx}(l)} \times A_{\mathrm{Rx}(k)}$ channel gain matrix between nodes $\operatorname{Tx}(l)$ and $\operatorname{Rx}(k)$. The interference at receiver $\operatorname{Rx}(k)$ 's $A_{\mathrm{Rx}(k)}$ antennas is $\left(\sum_{i=1}^{z_{l}[t]} \mathbf{u}_{l i}[t] s_{l i}[t]\right)^{\dagger} \mathbf{H}_{(l, k)}$. Receiver $\operatorname{Rx}(k)$ uses an $A_{\mathrm{Rx}(k)} \times 1$ receive weight vector $\mathbf{v}_{k j}[t]$ to receive data stream $j$ from transmitter $\operatorname{Tx}(k), 1 \leq j \leq z_{k}[t]$. The interference to data stream $j$ is

\footnotetext{
${ }^{2}$ Note that some other techniques such as multi-user detection [23, Chapter 7] and interference alignment [3] are not considered in this paper. This is because the maximum achievable rate region under these techniques remains unknown even for some simple topologies.

${ }^{3}$ Note that IC discussed in this paper is different from successive interference cancellation (SIC) in [23, Chapter 7]. SIC needs to decode interference before performing cancellation while IC does not require that interference be decoded. On the other hand, IC requires multiple antennas at each node while SIC does not.
} 


$$
\left(\sum_{i=1}^{z_{l}[t]} \mathbf{u}_{l i}[t] s_{l i}[t]\right)^{\dagger} \mathbf{H}_{(l, k)} \mathbf{v}_{k j}[t]=\sum_{i=1}^{z_{l}[t]}\left(\left(\mathbf{u}_{l i}[t]\right)^{\dagger} \mathbf{H}_{(l, k)} \mathbf{v}_{k j}[t]\right) \cdot s_{l i}[t] .
$$

In order to cancel the interference on each data stream $j$, the following IC constraints must be satisfied:

$$
\left(\mathbf{u}_{l i}[t]\right)^{\dagger} \mathbf{H}_{(l, k)} \mathbf{v}_{k j}[t]=0\left(1 \leq i \leq z_{l}[t], 1 \leq j \leq z_{k}[t]\right) .
$$

Based on the above discussion, a set of values for $\left(c_{1}, c_{2}, \cdots, c_{L}\right)$ is feasible if and only if we can find a feasible solution for all the transmit weight vectors and receive weight vectors in each time slot such that (1), (2), (3), and (4) hold. Note that although this matrix-based MIMO model is optimal in terms of identifying all feasible sets of values for $\left(c_{1}, c_{2}, \cdots, c_{L}\right)$ under SM and IC, its practical utility as an analysis tool is extremely limited. There are two troubling issues with this model. First, to obtain the rate region by (1), one needs to verify the feasibility of each set of values for $\left(z_{1}[t], z_{2}[t], \cdots, z_{L}[t]\right)$ by (2), (3), and (4). Note that each set of values for $\left(z_{1}[t], z_{2}[t], \cdots, z_{L}[t]\right)$ yields a different set of constraints and variables in (2), (3), and (4). Since one has to solve a different problem for each set of values for $\left(z_{1}[t], z_{2}[t], \cdots, z_{L}[t]\right)$, the number of problems that need to be solved is exponential with $L$. Second, verifying the feasibility of a given set of values for $\left(z_{1}[t], z_{2}[t], \cdots, z_{L}[t]\right)$ requires to solve a problem with a large number of bilinear equations (2), (3), and (4). Unlike linear equation systems, a general solution to bilinear equation systems remains unknown [9]. In Section IV-C, we will show that the high complexity of the matrix-based model even for a small three-link network.

\section{UNDERSTANDING DOF CONSUMPTION IN THE MATRIX-BASED MODEL}

Before we construct an optimal DoF-based link layer model for multi-hop MIMO networks, we must have a deep understanding on DoF consumption in the matrix-based model and obtain accurate results on the number of DoFs consumed by SM and IC, respectively.

\section{A. Basic Idea}

First, let's determine the total available DoFs for a transmit (or receive) weight vector at a node, which is associated for each data stream transmitted (or received) at this node. Initially, there is no constraint at a vector. Then each of its elements is undetermined and can be set arbitrarily. There is a feasible region (a space) that includes all possible values by such an unconstrained vector. The DoFs of this feasible region is equal to the number of elements in the unconstrained vector (or the number of antennas at the node). A vector's total available DoFs is defined as this initial DoFs.

We now show how DoFs are consumed when constraints are imposed on a vector. This can be illustrated by a substraction approach, i.e., first finding the vector's total available DoFs, then finding the vector's remaining DoFs (after constraints are imposed), and finally subtracting this remaining DoFs from the total available DoFs. Here, a vector's remaining DoFs is equal to the number of its linearly independent elements. As an example, consider a vector $\left[x_{1}, x_{2}, x_{3}, x_{4}, x_{5}\right]^{\dagger}$ with a total available DoFs of 5. Suppose we impose constraints $x_{1}=1$ and $x_{2}+x_{3}-x_{4}=0$ on this vector. Then the vector can be rewritten as $\left[1, x_{2}, x_{3}, x_{2}+x_{3}, x_{5}\right]^{\dagger}$. Given that the first element is set to 1 , there is no remaining DoF along this dimension. Since there is a linear relationship among the second, third, and fourth elements, the number of remaining DoFs of these elements is only 2 . For the fifth element $\left(x_{5}\right)$, its remaining DoF is 1 . Thus, the total number of remaining DoFs of this vector is 3 . As a result, the number of "consumed" DoFs (due to two imposed constraints) is $5-3=2$.

Alternatively, the number of consumed DoFs can be determined by directly analyzing the given constraints. Note that constraints considered in this paper are all linear constraints. A constraint can either set some element in a vector to a value (e.g., $x_{1}=1$ ) or set some linear relationship among multiple elements (e.g., $x_{2}+x_{3}-x_{4}=0$ ). In either case, the number of vector's DoFs is decreased by 1 . When there is no linear dependency among the given constraints, the number of consumed DoFs is equal to the number of constraints. As an example, the number of DoFs consumed by constraints $x_{1}=1$ and $x_{2}+x_{3}-x_{4}=0$ is 2 . When there is linear dependency among the given constraints, we should consider a subset of linearly independent constraints, and the number of consumed DoFs is equal to the number of linearly independent constraints. As an example, consider a vector $\left[x_{1}, x_{2}, x_{3}, x_{4}, x_{5}\right]^{\dagger}$ and the following three constraints.

$$
\begin{aligned}
2 x_{1} & =1 \\
2 x_{1}+x_{3}+5 x_{4} & =2 \\
6 x_{1}+x_{3}+5 x_{4} & =4
\end{aligned}
$$

Since (7) is a linear combination of (5) and (6), we have only 2 independent constraints. Thus, the number of DoFs consumed by these constraints is 2 . We summarize our discussion with the following lemma.

Lemma 1: The number of consumed DoFs of a vector due to a set of linear constraints among its elements is equal to the number of linearly independent constraints in this set.

Since the constraints for a transmit/receive vector are due to SM and IC, we will analyze each case in Sections III-B and III-C, respectively. In Section III-D, we show that the total consumed DoFs is the sum of DoFs consumed by SM and IC.

\section{B. DoF Consumption by SM}

We now analyze DoF consumption by SM in transmit and receive weight vectors in the matrix-based model. For a time slot $t$, we first consider a transmit weight vector $\mathbf{u}_{l i}$ at transmitter $\operatorname{Tx}(l)$. Note that for simplicity, we omit time slot $[t]$ in this section, e.g., using $\mathbf{u}_{l i}$ instead of $\mathbf{u}_{l i}[t]$. Under SM constraints (2) and (3), $\mathbf{u}_{l i}$ must satisfy the following constraints.

$$
\begin{aligned}
& \mathbf{u}_{l i}^{\dagger} \cdot\left(\mathbf{H}_{(l, l)} \mathbf{v}_{l i}\right)=1 \\
& \mathbf{u}_{l i}^{\dagger} \cdot\left(\mathbf{H}_{(l, l)} \mathbf{v}_{l j}\right)=0 \quad\left(1 \leq j \leq z_{l}, j \neq i\right)
\end{aligned}
$$

All the constraints in (8) and (9) are linear constraints. By Lemma 1, we need to analyze linear dependency among these 
constraints. It is easy to prove (by contradiction) that these $z_{l}$ constraints are all linearly independent [19]. Thus, we have the following lemma.

Lemma 2: For a link $l$ with $z_{l}$ data streams, the number of DoFs consumed by SM in each transmit weight vector $\mathbf{u}_{l i}$ at transmitter $T x(l)$ is $z_{l}$.

Lemma 2 can be explained intuitively as follows. The constraints in (8) and (9) ensure multiple orthogonal channels in spatial domain. Since each data stream should be transmitted in its own channel, the total number of channels required (corresponding to the number of consumed DoFs) is equal to the number of data streams $z_{l}$.

Now we consider a receive weight vector. Following the same token as for a transmit weight vector, we can prove the following lemma.

Lemma 3: For a link $l$ with $z_{l}$ data streams, the number of DoFs consumed by SM in each receive weight vector $\mathbf{v}_{l j}$ at receiver $R x(l)$ is $z_{l}$.

Lemma 3 can also be intuitively explained by that $z_{l}$ data streams will need $z_{l}$ channels in spatial domain and thus the number of consumed DoFs in each receive weight vector is $z_{l}$.

\section{DoF Consumption by IC}

We now analyze DoF consumption by IC in transmit and receive weight vectors in the matrix-based model. It turns out that unlike SM, DoFs consumed by IC only involve either a transmit weight vector or a receive weight vector, but not both. Now a new problem is: Which vector (transmit or receive weight vector) should consume its DoFs for IC? We find that for all transmit and receive weight vectors involved in IC, we can only determine one vector at a time and thus there is an order in this process when we go through all the vectors, i.e., which vector to consider first, second, etc. Further, once a particular order is given, one can find which vector should consume its DoFs for IC rather straightforward. Formal results for these findings are given in the rest of this section.

Based on the ordering concept, we can build a mathematical model by calculating DoF consumption for all vectors. However, such "vector-level" model will involve many variables and constraints and is cumbersome to work with. A good question to ask is: Can we simplify this vector-level model without any loss of rate region? We find that it is sufficient to consider a "node-level" ordering instead of vector-level ordering. Such node-level operation can significantly decrease the number of variables and constraints. Further, we prove that a model based on such node-level ordering can achieve the same rate region as that by a model based on vector-level ordering.

We organize this section as follows. In Section III-C1, we start with the simple two-link case. The ordering concept and the transition from vector-level ordering to node-level ordering are introduced here. We then present result for the general multi-link case in Section III-C2.

1) Two-Link Case: Let's consider the two-link case in Fig. 1, where the receiver $\operatorname{Rx}(k)$ of link $k$ is interfered by the transmitter $\operatorname{Tx}(l)$ of an interfering link $l$. Under the matrix-based model, some DoFs of transmit weight vectors at node $\operatorname{Tx}(l)$ or receive weight vectors at node $\operatorname{Rx}(k)$ will be consumed in IC constraints (4).

The Concept of Sequential Ordering. Before we analyze DoF consumption for IC, we reveal a sequential ordering among all transmit and receive weight vectors. We will show that such an order plays an important role for DoF consumption.

Note that by IC constraints (4), any pair of $\mathbf{u}_{l i}$ and $\mathbf{v}_{k j}$ are dependent. Depending on which vector is determined first, the other vector can be determined subsequently. That is, there is an ordering concept in determining the values of both vectors. For example, suppose the transmit weight vector $\mathbf{u}_{l i}$ is determined first, then receive weight vector $\mathbf{v}_{k j}$ can be determined via (4) subsequently. So the order in this example is first $\mathbf{u}_{l i}$ and then $\mathbf{v}_{k j}$. Without such an order, i.e., $\mathbf{u}_{l i}$ and $\mathbf{v}_{k j}$ being determined independently, it is unlikely that (4) can be satisfied.

Generalizing the above observation, we now consider all possible pairs of a $\mathbf{u}$ vector at transmitter $\operatorname{Tx}(l)$ and a $\mathbf{v}$ vector at receiver $\operatorname{Rx}(k)$ (total $z_{l} \cdot z_{k}$ pairs) in (4). Again, we must follow an order when determining all these vectors. Thus, we have a sequence of vectors. Denote such a sequential order as $\Pi$. For a transmit weight vector $\mathbf{u}_{l i}$, denote $\Pi_{\mathbf{u}_{l i}}$ the position of this vector in $\Pi$. Similarly, denote $\Pi_{\mathbf{v}_{k j}}$ the position of the receive weight vector $\mathbf{v}_{k j}$ in $\Pi$. Given that we have $z_{l}$ transmit weight vectors and $z_{k}$ receive weight vectors, the number of vectors in $\Pi$ is $z_{l}+z_{k}$. We summarize this observation as follows.

Observation 1: To cancel interference from transmitter $T x(l)$ 's $z_{l}$ data streams to receiver $R x(k)$ 's $z_{k}$ data streams, an order $\Pi$ must be followed when determining transmit weight vectors $\mathbf{u}_{l i}\left(1 \leq i \leq z_{l}\right)$ and receive weight vectors $\mathbf{v}_{k j}$ $\left(1 \leq j \leq z_{k}\right)$.

DoF Consumption Under A Sequential Order. Once we follow a sequential order when determining transmit/receive weight vectors, we suddenly have a new revelation in calculating DoF consumption in each vector by IC in the matrixbased model. This revelation is, in fact, the defining point of our contribution in uncovering DoF's fantastic property in IC.

We now analyze DoF consumption for a transmit weight vector and a receive weight vector, respectively.

Case A: Transmit weight vector $\mathbf{u}_{l i}$. By (4), vector $\mathbf{u}_{l i}$ must satisfy $\mathbf{u}_{l i}^{\dagger} \cdot\left(\mathbf{H}_{(l, k)} \mathbf{v}_{k j}\right)=0$ for $1 \leq j \leq z_{k}$. Let's begin by considering one constraint $\mathbf{u}_{l i}^{\dagger} \cdot\left(\mathbf{H}_{(l, k)} \mathbf{v}_{k j}\right)=0$ for a given $j$.

- If $\Pi_{\mathbf{v}_{k j}}<\Pi_{\mathbf{u}_{l i}}$, then by the time we consider $\mathbf{u}_{l i}$, vector $\mathbf{v}_{k j}$ has already been determined and we now have a linear constraint on $\mathbf{u}_{l i}$, which decreases $\mathbf{u}_{l i}$ 's DoFs by one.

- On the other hand, if $\Pi_{\mathbf{u}_{l i}}<\Pi_{\mathbf{v}_{k j}}$, i.e. $\mathbf{u}_{l i}$ is before $\mathbf{v}_{k j}$ in $\Pi$, it is not possible to impose any constraint on $\mathbf{u}_{l i}$ since $\mathbf{v}_{k j}$ is yet to be determined. Constraint $\mathbf{u}_{l i}^{\dagger}$. $\left(\mathbf{H}_{(l, k)} \mathbf{v}_{k j}\right)=0$ will be satisfied when we consider $\mathbf{v}_{k j}$ in the future. As a result, $\mathbf{u}_{l i}$ does not need to concern itself with this constraint and will thus not consume any DoF.

Thus, to analyze $\mathbf{u}_{l i}$ 's DoF consumption, we only need to 
consider the following constraints: $\mathbf{u}_{l i}^{\dagger} \cdot\left(\mathbf{H}_{(l, k)} \mathbf{v}_{k j}\right)=0$ for $1 \leq j \leq z_{k}$ and $\Pi_{\mathbf{v}_{k j}}<\Pi_{\mathbf{u}_{l i}}$. The number of these constraints is equal to the number of receive weight vectors that are placed before $\mathbf{u}_{l i}$ in $\Pi$. Further, we can assume that $\mathbf{H}_{(l, k)}$ is of full rank $^{4}$ and then verify that these constraints are all linearly independent. Therefore, the number of DoFs consumed by IC in $\mathbf{u}_{l i}$ is equal to the number of receive weight vectors that are placed before $\mathbf{u}_{l i}$ in $\Pi$.

Case B: Receive weight vector $\mathbf{v}_{k j}$. Following the same token, we have that the number of DoFs consumed for IC at a receive weight vector $\mathbf{v}_{k j}$ is equal to the number of transmit weight vectors that are placed before $\mathbf{v}_{k j}$ in $\Pi$.

The following lemma summarizes our discussion. Its proof can be found in [19].

Lemma 4: Consider the interference from transmitter $T x(l)$ 's $z_{l}$ data streams to receiver $R x(k)$ 's $z_{k}$ data streams. Based on IC constraint (4) in the matrix-based model, we have (i) for a transmit weight vector $\mathbf{u}_{l i}$, the number of DoFs consumed by $I C$ in $\mathbf{u}_{l i}$ is equal to the number of receive weight vectors at $R x(k)$ that are placed before $\mathbf{u}_{l i}$ in $\Pi$; (ii) for a receive weight vector $\mathbf{v}_{k j}$, the number of DoFs consumed by IC in $\mathbf{v}_{k j}$ is equal to the number of transmit weight vectors at $T x(l)$ that are placed before $\mathbf{v}_{k j}$ in $\Pi$.

Since different sequential order $\Pi$ will yield different DoF consumption in a transmit/receive weight vector, such order $\Pi$ should be subject to optimization in a particular problem.

From Vector-Level to Node-Level. The sequential order in Lemma 4 is on vector level. A model based on such vectorlevel ordering would have too many variables and constraints, which is cumbersome to work with. To simplify the model, we now consider a special vector-level ordering, where we visit each node following some sequential order $\pi$, and once we are at a node, we can determine all the vectors at this node following an arbitrary order. For this special vector-level order, it is easy to verify that, by Lemma 4, all vectors at the same node will have the same DoF consumption and the order among the vectors at the same node does not affect DoF consumption.

As a result of this finding, we may consider a "nodelevel" ordering $\pi$ among the nodes in the network. For a transmitter $\operatorname{Tx}(l)$, denote $\pi_{\mathrm{Tx}(l)}$ the position of this node in $\pi$. Similarly, denote $\pi_{\mathrm{Rx}(k)}$ the position of a receiver $\operatorname{Rx}(k)$ in $\pi$. By Lemma 4, it is easy to prove the following lemma.

Lemma 5: Consider the interference from transmitter $T x(l)$ 's $z_{l}$ data streams to receiver $R x(k)$ 's $z_{k}$ data streams. Based on IC constraint (4) in the matrix-based model, we have (i) if $\pi_{T_{x}(l)}>\pi_{R x}(k)$, then the number of DoFs consumed by IC are $z_{k}$ and 0 at $T x(l)$ and $R x(k)$, respectively; (ii) if $\pi_{T x(l)}<\pi_{R x}(k)$, then the number of DoFs consumed by IC are 0 and $z_{l}$ at $T x(l)$ and $R x(k)$, respectively.

For the rate region achieved by node-level ordering, we have the following lemma.

Lemma 6: For the two-link case, the achievable rate region by the matrix-based model with a node-level ordering is the

\footnotetext{
${ }^{4}$ This holds when the scattering in the environment is sufficiently rich, e.g., an urban environment.
}

$$
\begin{array}{rllll}
\text { A vector-level ordering: } & \boldsymbol{u}_{l 2} & \boldsymbol{v}_{k l} & \boldsymbol{u}_{l l} & \boldsymbol{v}_{k 2} \\
\text { The constructed vector-level ordering: } & \boldsymbol{u}_{l 2} & \boldsymbol{u}_{l l} & \boldsymbol{v}_{k l} & \boldsymbol{v}_{k 2} \\
\text { The constructed node-level ordering: } & \mathrm{Tx}_{l} & \multicolumn{2}{c}{\mathrm{Rx}_{k}}
\end{array}
$$

Fig. 2. An example showing the transition from vector-level ordering to node-level ordering.

same as that under the matrix-based model with a vector-level ordering.

To prove this lemma, it is sufficient to show that for any feasible rates $\left(z_{l}, z_{k}\right)$ that can be achieved by the matrix-based model with a vector-level ordering $\Pi$, we can construct a nodelevel ordering $\pi$ to achieve the same rates $\left(z_{l}, z_{k}\right)$. Let's see the following example.

Example 1: Consider two links $l$ and $k$ with $z_{l}=2$ and $z_{k}=2$ data streams, respectively. Assume there are 4 antennas on each of the transmitting and receiving nodes. Then the total available DoFs in each transmit or receive weight vector is 4 . Suppose the vector-level ordering $\Pi$ is $\mathbf{u}_{l 2}, \mathbf{v}_{k 1}, \mathbf{u}_{l 1}, \mathbf{v}_{k 2}$ (see the first line in Fig. 2). We now show how to construct a nodelevel ordering $\pi$ based on $\Pi$ such that data rates $z_{l}=2$ and $z_{k}=2$ remain feasible.

Denote $D_{o} F_{l}\left(\mathbf{v}_{k j}\right)$ the DoFs consumed by IC in receive weight vector $\mathbf{v}_{k j}$. Based on Lemma 4, we have $D o F_{l}\left(\mathbf{v}_{k 1}\right)=$ 1 and $D o F_{l}\left(\mathbf{v}_{k 2}\right)=2$. Since $D o F_{l}\left(\mathbf{v}_{k 1}\right)<D o F_{l}\left(\mathbf{v}_{k 2}\right), \mathbf{v}_{k 2}$ is the bottleneck receive weight vector at receiver $\operatorname{Rx}(k)$, which has the smallest remaining DoFs $(4-2=2)$ for SM. Similarly, denote $D o F_{k}\left(\mathbf{u}_{l i}\right)$ the DoFs consumed for IC at transmit weight vector $\mathbf{u}_{l i}$. Based on Lemma 4 , we have $D o F_{k}\left(\mathbf{u}_{l 2}\right)=0$, and $D o F_{k}\left(\mathbf{u}_{l 1}\right)=1$. We can see that $\mathbf{u}_{l 1}$ is the bottleneck transmit weight vector at transmitter $\operatorname{Tx}(l)$, which has the smallest remaining DoFs $(4-1=3)$ for SM.

To construct a node-level ordering $\pi$, we first re-order the vectors as $\mathbf{u}_{l 2}, \mathbf{u}_{l 1}, \mathbf{v}_{k 1}, \mathbf{v}_{k 2}$, where the first two vectors are the transmit weight vectors and the remaining two vectors are the receive weight vectors (see the second line in Fig. 2). Based on Lemma 4, we have $D o F_{l}\left(\mathbf{v}_{k 1}\right)=D o F_{l}\left(\mathbf{v}_{k 2}\right)=2$ under this new order. We find that although $D_{o} F_{l}\left(\mathbf{v}_{k 1}\right)$ is increased from 1 to $2, \mathbf{v}_{k 2}$ remains a bottleneck receive weight vector. Thus, $z_{k}=2$ is still feasible under this new ordering. Similarly, based on Lemma 4, we have $D o F_{k}\left(\mathbf{u}_{l 2}\right)=D o F_{k}\left(\mathbf{u}_{l 1}\right)=0$ under this new order. Since $D o F_{k}\left(\mathbf{u}_{l 1}\right)$ is decreased from 1 to 0 , this vector has more DoFs remaining than in $\Pi$. Thus, $z_{l}=2$ is still feasible under this new order. In summary, rates $\left(z_{l}, z_{k}\right)$ remain feasible under the new ordering.

For this new vector-level order, if we group the first two transmit weight vectors at transmitter $\operatorname{Tx}(l)$ and the rest two receive weight vectors at receiver $\operatorname{Rx}(k)$, then we have a nodelevel order (see the third line in Fig. 2). Note that for this node-level order, the DoF consumption in each vector at the same node is identical (i.e., $D o F_{l}\left(\mathbf{v}_{k 1}\right)=D o F_{l}\left(\mathbf{v}_{k 2}\right)=2$ and $\left.D o F_{k}\left(\mathbf{u}_{l 2}\right)=D o F_{k}\left(\mathbf{u}_{l 1}\right)=0\right)$. We call this node level order $\pi$, which can achieve the same rates $\left(z_{l}, z_{k}\right)$ as in $\Pi$. $\square$

A formal proof of Lemma 6 based on the idea in Example 1 can be found in [19].

2) Multi-Link Case: For the general multi-link case, we need to analyze two cases: (i) DoF consumption at a receiver 


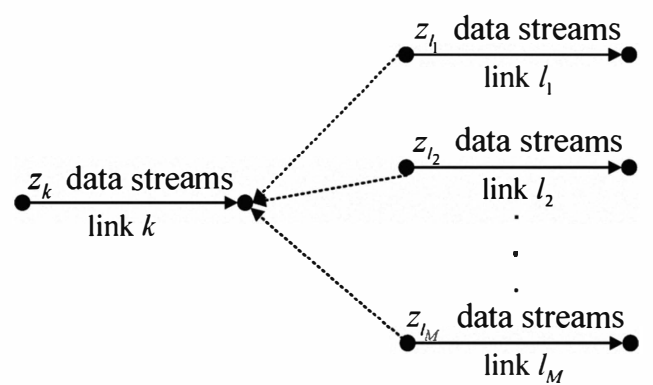

(a) One link $(k)$ is interfered by multiple links $\left(l_{1}, l_{2}, \cdots, l_{M}\right)$.

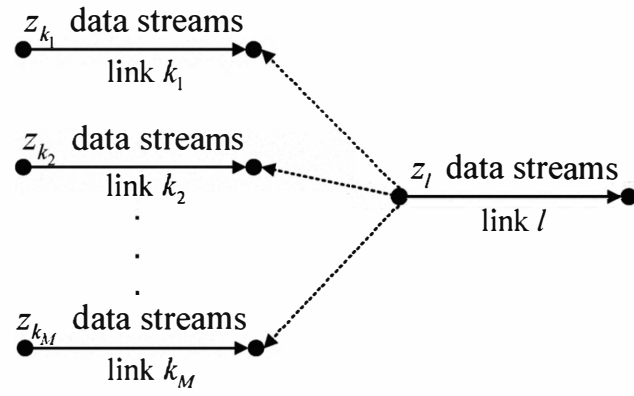

(b) One link ( $l)$ interferes multiple links $\left(k_{1}, k_{2}, \cdots, k_{M}\right)$.

Fig. 3. The multi-link case.

that is being interfered by multiple other links (see Fig. 3(a)), and (ii) DoF consumption at a transmitter that interferes multiple other links (see Fig. 3(b)). The analysis follows the same token as the two-link case. That is, we first reveal a sequential ordering among all transmit and receive weight vectors. Then we can analyze DoF consumption for each vector. We further show that such a vector-level ordering can be simplified as a node-level ordering without any loss on the achievable rate region. Therefore, we have the following theorem. Its proof can be found in [19].

Theorem 1: For the scenario in Fig. 3(a), the number of DoFs consumed by IC at the receiver $R x(k)$ is $\sum_{m=1}^{M}\left(z_{l_{m}}\right.$. $\left.1^{+}\left\{\pi_{T_{x}\left(l_{m}\right)}<\pi_{R x}(k)\right\}\right)$.

For the scenario in Fig. 3(b), the number of DoFs consumed by IC at the transmitter $T x(l)$ is $\sum_{m=1}^{M}\left(z_{l_{m}} \cdot 1^{+}\left\{\pi_{T_{x}\left(l_{m}\right)}<\right.\right.$ $\left.\left.\pi_{R x}(k)\right\}\right)$.

\section{Total Consumed DoFs}

We have analyzed DoF consumptions by SM and IC in Sections III-B and III-C, respectively. The remaining question becomes: Is the total number of consumed DoFs a simple sum of those by SM and IC? The answer is yes if and only if there is no linear dependency between the set of SM and IC constraints. We prove that there is no such linear dependency in [19]. Thus, we have the following lemma.

Lemma 7: The total consumed DoFs in the matrix-based model is the sum of DoFs consumed by SM and IC.

\section{AN Optimal DoF-BASEd Model}

\section{A. Mathematical Modeling}

Based on the results in the previous section, we are now ready to develop an optimal link layer model for a multihop MIMO network. We have the following four sets of constraints.

Half-Duplex Constraint. Due to the half-duplex property, a node cannot be the transmitter of one link and the receiver of another link in the same time slot. We use a binary variable $x_{i}[t], 1 \leq i \leq N$ and $1 \leq t \leq T$, to indicate whether node $i$ is a transmitter for some link in time slot $t$. That is, if node $i$ is a transmitter in time slot $t$, then $x_{i}[t]=1$, otherwise $x_{i}[t]=0$. We use another binary variable $y_{i}[t], 1 \leq i \leq N$ and $1 \leq t \leq$ $T$, to indicate whether node $i$ is a receiver for some link in time slot $t$. Then the half-duplex property can be modeled as

$$
x_{i}[t]+y_{i}[t] \leq 1 \quad(1 \leq i \leq N, 1 \leq t \leq T) .
$$

Constraints for Node Activity. Denote $\mathcal{L}_{i}^{\text {in }}$ and $\mathcal{L}_{i}^{\text {out }}$ the set of possible incoming and outgoing links at node $i$, respectively. Note that a node can be the transmitter of multiple links or the receiver of multiple links in the same time slot by IC.

If node $i$ is not an active transmitter, then we have $\sum_{l \in \mathcal{L}_{i}^{\text {out }}} z_{l}[t]=0$. Otherwise, by the fact that the total available DoFs of a vector at node $i$ is equal to $A_{i}$ (the number of antennas at node $i$ ), we have $1 \leq \sum_{l \in \mathcal{L}_{i}^{\text {out }}} z_{l}[t] \leq A_{i}$ by Lemmas 2 . These two cases can be formulated by the following constraint.

$$
x_{i}[t] \leq \sum_{l \in \mathcal{L}_{i}^{\text {out }}} z_{l}[t] \leq A_{i} \cdot x_{i}[t] \quad(1 \leq i \leq N, 1 \leq t \leq T) .
$$

Similarly, considering whether or not node $i$ is an active receiver, we have

$$
y_{i}[t] \leq \sum_{l \in \mathcal{L}_{i}^{\text {in }}} z_{l}[t] \leq A_{i} \cdot y_{i}[t] \quad(1 \leq i \leq N, 1 \leq t \leq T) .
$$

Ordering Constraints. For any order $\pi[t]$, we have

$$
1 \leq \pi_{i}[t] \leq N \quad(1 \leq i \leq N, 1 \leq t \leq T) .
$$

To model the "relative" ordering between any two nodes $i$ and $j$ in $\pi[t]$, we use a binary variable $\theta_{j i}[t]$ and define it as follows: $\theta_{j i}[t]=1$ if node $i$ is after node $j$ in $\pi[t]$ (not necessarily consecutive) and 0 otherwise. It is easy to verify that the following relationships hold among $\pi_{i}[t], \pi_{j}[t]$, and $\theta_{j i}[t]$.

$$
\begin{aligned}
& \pi_{i}[t]-N \cdot \theta_{j i}[t]+1 \leq \pi_{j}[t] \leq \pi_{i}[t]-N \cdot \theta_{j i}[t]+N-1 \\
&\left(1 \leq i \leq N, j \in \mathcal{I}_{i}, 1 \leq t \leq T\right),
\end{aligned}
$$

where $\mathcal{I}_{i}$ is the set of nodes within node $i$ 's interference range.

DoF Consumption Constraints. It is clear that the total consumed DoFs at a node cannot exceed its total available DoFs (or the number of antennas at this node). Thus, if node $i$ is a transmitter, then we have $\sum_{l \in \mathcal{L}_{i}^{\text {out }}} z_{l}[t]+\sum_{j \in \mathcal{I}_{i}} \theta_{j i}[t] \sum_{k \in \mathcal{L}_{j}^{\text {in }}}^{\mathrm{Tx}(k) \neq i} z_{k}[t] \leq A_{i}$ by Lemma 2 
and Theorem 1. Otherwise, if node $i$ is not a transmitter, then $\sum_{l \in \mathcal{L}_{i}^{\text {out }}} z_{l}[t]=0$ and there is no constraint on $\sum_{j \in \mathcal{I}_{i}} \theta_{j i}[t] \sum_{k \in \mathcal{L}_{j}^{\text {in }}}^{\operatorname{Tx}(k) \neq i} z_{k}[t]$. To develop one constraint for both cases, we introduce a large constant $B_{i}$ (e.g., setting $B_{i}=\sum_{j \in \mathcal{I}_{i}} A_{j}$ ) to ensure that $B_{i}$ is an upper bound of $\sum_{j \in \mathcal{I}_{i}} \theta_{j i}[t] \sum_{k \in \mathcal{L}_{j}^{\text {in }}}^{\mathrm{Tx}(k) \neq i} z_{k}[t]$. Then we have

$$
\begin{array}{r}
\sum_{l \in \mathcal{L}_{i}^{\text {out }}} z_{l}[t]+\sum_{j \in \mathcal{I}_{i}} \theta_{j i}[t] \sum_{k \in \mathcal{L}_{j}^{\text {in }}}^{\mathrm{Tx}(k) \neq i} z_{k}[t] \leq A_{i} x_{i}[t]+\left(1-x_{i}[t]\right) B_{i} \\
(1 \leq i \leq N, 1 \leq t \leq T) .
\end{array}
$$

Now we consider the case of whether or not node $i$ is a receiver. Following the same token, we have

$$
\begin{aligned}
\sum_{k \in \mathcal{L}_{i}^{\text {in }}} z_{k}[t]+\sum_{j \in \mathcal{I}_{i}} \theta_{j i}[t] \sum_{l \in \mathcal{L}_{j}^{\text {out }}}^{\mathrm{Rx}(l) \neq i} z_{[}[t] & \leq A_{i} x_{i}[t]+\left(1-y_{i}[t]\right) B_{i} \\
& (1 \leq i \leq N, 1 \leq t \leq T) .
\end{aligned}
$$

Note that $z_{l}[t]$ is the number of data streams on link $l$ in time slot $t$. By (1), we can calculate the achievable rate $c_{l}$, which is the average of $z_{l}[t]$ over all $T$ time slots. Thus, a model for the rate region of $\left(c_{1}, c_{2}, \cdots, c_{L}\right)$ includes constraints (1), (10)-(16). Note that there is no matrix representation involved in this DoF-based model. Further, nonlinear constraints in (15) and (16) can be reformulated as equivalent linear constraints [19]. Therefore, we have a linear link layer model under SM and IC for any MIMO network topology.

\section{B. Complexity and Performance Comparison}

Complexity Comparison. We now show that, comparing to the matrix-based model (1)-(4), our model is much simpler. First, note that for the matrix-based model, the set of constraints and variables in (2), (3), and (4) depends on the set of values for $\left(z_{1}[t], z_{2}[t], \cdots, z_{L}[t]\right)$. Since we have to solve one problem for each set of values for $\left(z_{1}[t], z_{2}[t], \cdots, z_{L}[t]\right)$, the number of problems that need to be solved is exponential with $L$. Second, in the matrix-based model, verifying the feasibility of a given set of $\left(z_{1}[t], z_{2}[t], \cdots, z_{L}[t]\right)$ requires to solve a large number of bilinear equations (2), (3), and (4), which are very difficult.

In our DoF-based model, the set of constraints and variables does not depend on the set of values for $\left(z_{1}[t]\right.$, $\left.z_{2}[t], \cdots, z_{L}[t]\right)$. Thus, we only need to solve one problem. Moreover, all constraints in this problem are linear.

Achievable Rate Region. For the performance of our model, we have the following theorem.

Theorem 2: Our DoF-based link layer model for multi-hop MIMO networks can achieve the same rate region as that by the matrix-based model.

We give a sketch of the proof here. Since rates $\left(c_{1}, c_{2}\right.$, $\left.\cdots, c_{L}\right)$ are calculated by $\left(z_{1}[t], z_{2}[t], \cdots, z_{L}[t]\right)$ in (1), to prove that both models have the same rate region, it is sufficient to show that both models have the same rate region $\left(z_{1}[t], z_{2}[t], \cdots, z_{L}[t]\right)$ for each time slot $t$. That is,

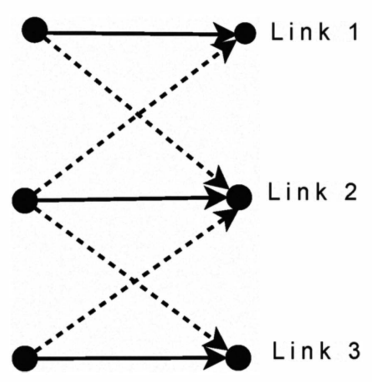

Fig. 4. A three-link network.

we will show that any feasible $\left(z_{1}[t], z_{2}[t], \cdots, z_{L}[t]\right)$ under the matrix-based model is also feasible under our DoFbased model, and vice versa. Note that for any feasible $\left(z_{1}[t], z_{2}[t], \cdots, z_{L}[t]\right)$ under the matrix-based model, we have a solution of all transmit and receive weight vectors to satisfy SM and IC constraints. Based on results in Section III, we are able to identify DoF consumption for SM and IC at each node by Lemmas 2 and 3 and Theorem 1, respectively. Thus, we have a corresponding solution for DoF consumption. This solution is also feasible under our DoF-based model since our model is built upon the same Lemmas 2 and 3 and Theorem 1 . On the other hand, for any feasible $\left(z_{1}[t], z_{2}[t], \cdots, z_{L}[t]\right)$ under our DoF-based model, we have a solution giving an order among all the nodes in the network and DoF consumption at all the nodes. Based on the discussion in Section III, this solution corresponds to an order to determine all transmit and receive weight vectors and the set of SM and IC constraints that each vector must satisfy. Since by (15) and (16), our DoF-based model can guarantee that the number of imposed constraints on a vector is no more than the number of its elements, we can prove that there is always a feasible solution for each vector. Thus, we have a corresponding feasible solution on vectors. As a result, the same $\left(z_{1}[t], z_{2}[t], \cdots, z_{L}[t]\right)$ is feasible under the matrix-based model. Theorem 2 is proved by combining both results.

\section{Numerical Results}

We now compare the rate region and complexity between our DoF-based model and the matrix-based model for a threelink network. We will show that they achieve the same rate region while our DoF-based model incurs significantly less complexity. We will also show that the rate region under a previous DoF-based model, CiM [7], is smaller than that under our model.

Fig. 4 shows the topology of three active links, where links 1 and 2 are interfering with each other and links 2 and 3 are interfering with each other. Suppose that the number of antennas at both the transmitter and the receiver of link 1 are six, the number of antennas at both the transmitter and the receiver of link 2 are four, and the number of antennas at both the transmitter and the receiver of link 3 are two. 
TABLE I

COMPLEXITY COMPARISON BETWEEN THE MATRIX-BASED MODEL AND OUR DOF-BASED MODEL FOR A THREE-LINK NETWORK.

\begin{tabular}{|c|c|c|}
\hline & Matrix-based model & DoF-based model \\
\hline Number of Problems & 105 & 1 \\
\hline Type of Problems & Bilinear problems & Linear program \\
\hline
\end{tabular}

We now show how to obtain the rate region for this threelink network under the matrix-based model and our DoF-based model.

- Under the matrix-based model, to compute $\left(c_{1}, c_{2}, c_{3}\right)$ by (1), we need to verify the feasibility of each set of values for $\left(z_{1}[t], z_{2}[t], z_{3}[t]\right)$. We use $\left(z_{1}[t], z_{2}[t], z_{3}[t]\right)=$ $(3,1,1)$ as an example. We need to check whether we can find three $6 \times 1$ transmit weight vectors $\mathbf{u}_{11}[t], \mathbf{u}_{12}[t]$, and $\mathbf{u}_{13}[t]$ at $\operatorname{Tx}(1)$, three $6 \times 1$ receive weight vectors $\mathbf{v}_{11}[t], \mathbf{v}_{12}[t]$, and $\mathbf{v}_{13}[t]$ at $\operatorname{Rx}(1)$, one $4 \times 1$ transmit weight vectors $\mathbf{u}_{21}[t]$ at $\operatorname{Tx}(2)$, one $4 \times 1$ receive weight vectors $\mathbf{v}_{21}[t]$ at $\operatorname{Rx}(2)$, one $2 \times 1$ transmit weight vectors $\mathbf{u}_{31}[t]$ at $\operatorname{Tx}(3)$, and one $2 \times 1$ receive weight vectors $\mathbf{v}_{31}[t]$ at $\mathrm{Rx}(3)$ such that bilinear constraints (2), (3), and (4) hold. There are $\left(z_{1}[t]\right)^{2}+\left(z_{2}[t]\right)^{2}+$ $\left(z_{3}[t]\right)^{2}+2 z_{1}[t] z_{2}[t]+2 z_{2}[t] z_{3}[t]=19$ constraints and $12 z_{1}[t]+8 z_{2}[t]+4 z_{3}[t]=48$ variables (note that a $6 \times 1$ vector has six variables) in these bilinear constraints. Since a general solution to bilinear equation systems remains unknown [9], it can only be solved via exhaustive search. We finally find vectors to satisfy all the constraints and thus $(3,1,1)$ is feasible. This verification process for a single set of $\left(z_{1}[t], z_{2}[t], z_{3}[t]\right)=(3,1,1)$ is already very complex.

Now suppose we want to check the feasibility of $\left(z_{1}[t], z_{2}[t], z_{3}[t]\right)=(1,2,1)$. We have a problem of 14 bilinear constraints with 32 variables. Thus, the problem for different set of $\left(z_{1}[t], z_{2}[t], z_{3}[t]\right)$ is different.

Since $z_{1}[t] \in\{0,1,2,3,4,5,6\}, z_{2}[t] \in\{0,1,2,3,4\}$, and $z_{3}[t] \in\{0,1,2\}$, we need to solve $7 \times 5 \times 3=$ 105 bilinear problems to determine feasibility of each set of $\left(z_{1}[t], z_{2}[t], z_{3}[t]\right)$. Then we obtain the rate region in Fig. 5.

- Now we compute the rate region under our DoF-based model. Instead of verifying each set of values for $\left(z_{1}[t], z_{2}[t], z_{3}[t]\right)$ by solving 105 different problems as in the matrix-based model, our DoF-based model only needs to solve one linear problem and obtain all possible sets of $\left(z_{1}[t], z_{2}[t], z_{3}[t]\right)$ values. The problem we are solving now is to find $z_{l}[t]$ on each link $l$ and an order $\pi[t]$ such that all constraints in Section IV-A hold. Note that constraints (15) and (16) can be reformulated as equivalent linear constraints [19]. After solving this linear program, we have $z_{3}[t] \leq 2, z_{1}[t]+z_{2}[t] \leq 6, z_{2}[t]+z_{3}[t] \leq 4$. This rate region is shown in Fig. 5, which is the same as that by the matrix-based model.

Table I summarizes the above discussion. Although the rate regions by both models are identical, our DoF-based model can be solved with a much lower complexity because (i) it only requires to solve one problem, instead of many problems under the matrix-based model, and (ii) the problem under our

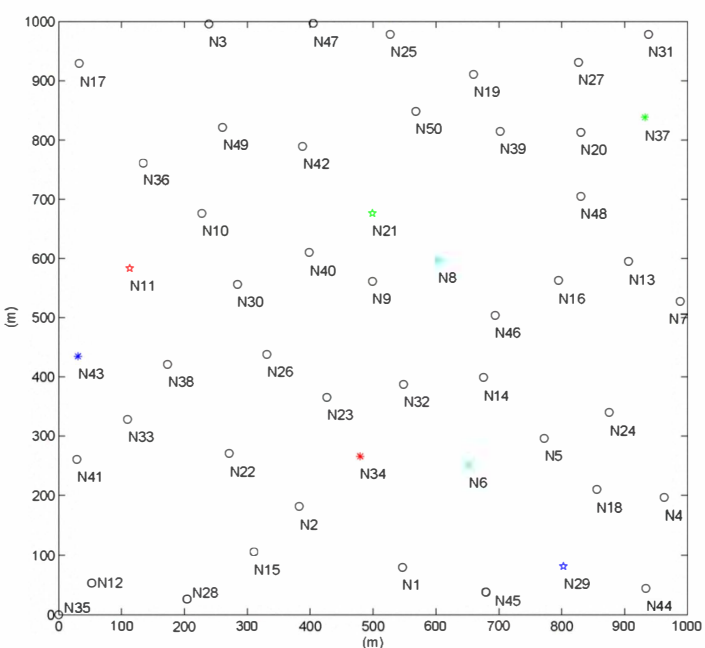

Fig. 6. A 50-node multi-hop MIMO network.

TABLE II

NODE ORDERING RESULTS IN EACH TIME SLOT OF A FRAME.

\begin{tabular}{|c|c|c|c|c|}
\hline$i$ & $\pi_{i}[1]$ & $\pi_{i}[2]$ & $\pi_{i}[3]$ & $\pi_{i}[4]$ \\
\hline 1 & 7 & 13 & 8 & 11 \\
\hline 2 & 11 & 8 & 10 & 8 \\
\hline 6 & 12 & 7 & 7 & 3 \\
\hline 8 & 2 & 1 & 3 & 9 \\
\hline 11 & 9 & 12 & 5 & 12 \\
\hline 14 & 8 & 9 & 11 & 6 \\
\hline 19 & 3 & 14 & 14 & 10 \\
\hline 21 & 6 & 6 & 4 & 4 \\
\hline 22 & 13 & 11 & 9 & 7 \\
\hline 26 & 10 & 5 & 12 & 13 \\
\hline 29 & 14 & 10 & 2 & 2 \\
\hline 34 & 5 & 3 & 6 & 5 \\
\hline 37 & 1 & 4 & 1 & 1 \\
\hline 43 & 4 & 2 & 13 & 14 \\
\hline
\end{tabular}

DoF-based model is a linear problem while the problems under the matrix-based model are bilinear problems.

We also show the rate region under $\mathrm{CiM}$ in Fig. 5, which is the inside tetrahedron. The ratio between the rate regions under CiM and our model is $\frac{3}{10}$.

\section{An Application of Our Model}

In this section, we show how to apply our link layer model to solve a cross-layer throughput maximization problem for a multi-hop MIMO network, which is our main purpose of developing such a model.

As a case study, we consider how to maximize, say, the sum of weighted rates for a set of sessions $\mathcal{F}$ in a multi-hop MIMO networks. For each session $f \in \mathcal{F}$, denote $r(f)$ the rate of session $f$ and $w(f)$ the weight of session $f$. Denote $r_{l}(f)$ the amount of rate on link $l$ attributed to session $f$. At the network layer, minimum-hop routing is employed. Since the total data rate on any link cannot exceed its achievable rate, we have

$$
\sum_{f \in \mathcal{F}} r_{l}(f) \leq c_{l} \quad(1 \leq l \leq L) .
$$

The problem is maximizing $\sum_{f \in \mathcal{F}} w(f) \cdot r(f)$, subject to (1) and all the constraints in Section IV-A. 


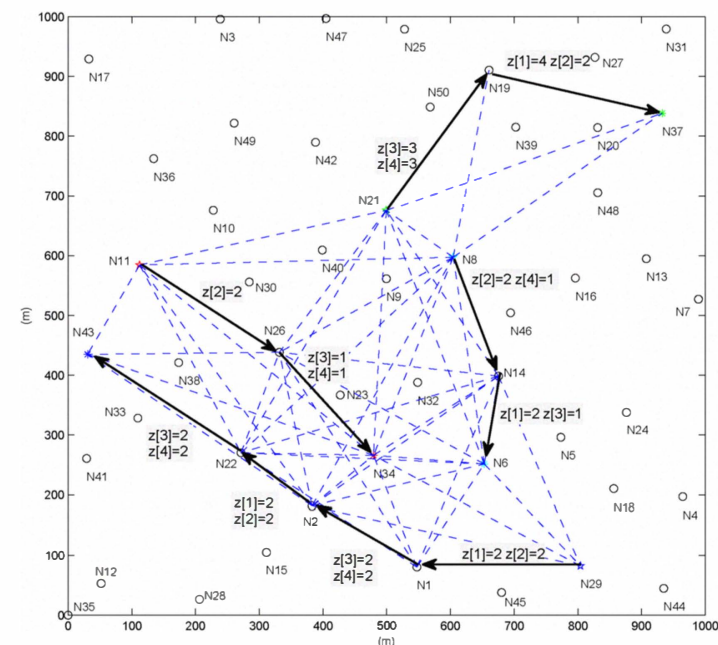

Fig. 7. Scheduling result on each link.

A multi-hop MIMO network consisting of 50 nodes is shown in Fig. 6. Each node in the network is equipped with four antennas. There are four sessions in the network: N11 to $\mathrm{N} 34, \mathrm{~N} 21$ to $\mathrm{N} 37, \mathrm{~N} 29$ to $\mathrm{N} 43, \mathrm{~N} 8$ to N6 with weights $0.7,0.4,0.8$ and 0.9 , respectively. Suppose that there are $T=4$ time slots in each time frame. This cross-layer optimization problem can be solved by CPLEX. We have the optimal objective value 2.425 , the optimal node ordering in Table $\mathrm{II}^{5}$, and the scheduling solutions in Fig. 7. As an example, the shaded box next to link N8 $\rightarrow \mathrm{N} 14$ contains $z[2]=2, z[4]=1$, which means that there are two data streams on this link in time slot 2 and one data stream on this link in time slot 4 . In other time slots (time slots 1 and 3 ), this link is not active.

\section{CONCLUSION}

The original matrix-based MIMO model is too complex for network level analysis and cross-layer optimization. Simple models based on DoF abstraction only require numeric computations on DoFs for SM and IC and thus offer significant advantages over the matrix-based model. However, existing DoF-based models are based on sufficient conditions on DoFs and data streams and cannot guarantee the same rate region as that under the matrix-based model. In this paper, we developed an optimal DoF-based model for a multi-hop MIMO network under SM and IC. It retains the same simplicity as previous DoF-based models while offering the same achievable rate region as that by the matrix-based model. Our optimal DoFbased model can be used as a reference model for theoretical research on multi-hop MIMO networks.

\section{ACKNOWLEDGMENTS}

This work was supported in part by the NSF under Grant CNS-0721421 and the ONR under Grant N00014-08-1-0084.

\section{REFERENCES}

[1] R. Bhatia and L. Li, "Throughput optimization of wireless mesh networks with MIMO links," in Proc. IEEE INFOCOM, pp. 2326-2330, Anchorage, AK, May 6-12, 2007.

${ }^{5}$ Only those active nodes (involved in multi-hop routing) are shown in Table II.
[2] E. Biglieri, R. Calderbank, A. Constantinides, A. Goldsmith, A. Paulraj, and H.V. Poor, MIMO Wireless Communications, Cambridge University Press, Jan. 2007.

[3] V. R. Cadambe and S.A. Jafar, "Interference alignment and the degrees of freedom for the $K$-user interference channel," IEEE Transactions on Information Theory, vol. 54, issue 8, pp. 3425-3441, Aug. 2008.

[4] L.-U. Choi and R.D. Murch, "A transmit preprocessing technique for multiuser MIMO systems using a decomposition approach," IEEE Transactions on Wireless Communications, vol. 3, no. 1, pp. 20-24, Jan. 2004.

[5] S. Chu and X. Wang, "Opportunistic and cooperative spatial multiplexing in MIMO ad hoc networks," in Proc. ACM MobiHoc, pp. 63-72, Hong Kong, China, May 26-30, 2008.

[6] A. Goldsmith, S.A. Jafar, N. Jindal, and S. Vishwanath, "Capacity limits of MIMO channels," IEEE Journal of Selected Areas in Communications, vol. 21, no. 1, pp. 684-702, June 2003.

[7] B. Hamdaoui and K.G. Shin, "Characterization and analysis of multi-hop wireless MIMO network throughput," in Proc. ACM MobiHoc, pp. 120129, Montreal, Quebec, Canada, Sep. 9-14, 2007.

[8] IEEE Transactions on Information Theory-Special Issue on Space-Time Transmission, Reception, Coding and Signal Processing, Guest Editors: B.M. Hochwald, G. Caire, B. Hassibi, and T.L. Marzetta, vol. 49, no. 10, Oct. 2003.

[9] C.R. Johnson and J.A. Link, "Solution theory for complete bilinear systems of equations," Numerical Linear Algebra with Applications, vol. 16, issue 11-12, pp. 929-934, Nov.-Dec. 2009.

[10] IEEE Journal on Selected Areas in Communications-Special Issue on MIMO Systems and Applications: Part I and Part II, Guest Editors: M. Shafi, D. Gesbert, D. Shiu, and P.J. Smith, vol. 21, no. 3 \& 5, April \& June 2003.

[11] IEEE Journal on Selected Areas in Communications-Special Issue on MIMO Systems and Applications: Field Experience, Practical Aspects, Limitations and Challenges, Guest Editors: M. Shafi, H. Huang, A. Hottinen, P.J. Smith, and R.A. Valenzuela, vol. 26, no. 6, Aug. 2008.

[12] S.-J. Kim, X. Wang, and M. Madihian, "Cross-layer design of wireless multihop backhaul networks with multiantenna beamforming," IEEE Transactions on Mobile Computing, vol. 6, no. 11, pp. 1259-1269, Nov. 2007.

[13] J. Liu, Y. Shi, and Y.T. Hou, "A tractable and accurate cross-layer model for multi-hop MIMO networks," in Proc. IEEE INFOCOM, 9 pages, San Diego, CA, March 15-19, 2010.

[14] J. Mundarath, P. Ramanathan, and B.D. Van Veen, "Exploiting spatial multiplexing and reuse in multi-antenna wireless ad hoc networks," Elsevier Ad Hoc Networks, vol. 7, no. 2, pp. 281-293, March 2009.

[15] A.J. Paulraj, D.A. Gore, R.U. Nabar, and H. Bolcskei, "An overview of MIMO communications - A key to gigabit wireless," in Proc. IEEE, vol. 92, no. 2, pp. 198-218, Feb. 2004.

[16] M. Park, R. Heath, and S. Nettles, "Improving throughput and fairness of MIMO ad hoc networks using antenna selection diversity," in Proc. IEEE GLOBECOM, pp. 3363-3367, Dallas, TX, Nov. 29-Dec.3, 2004.

[17] J.-S. Park, A. Nandan, M. Gerla, and H. Lee, "SPACE-MAC: Enabling spatial reuse using MIMO channel-aware MAC," in Proc. IEEE ICC, pp. 3642-3646, Seoul, Korea, May 16-20, 2005.

[18] M. Park, S-H. Choi, and S. Nettles, "Cross-layer MAC design for wireless networks using MIMO," in Proc. IEEE GLOBECOM, pp. 2870 2874, St Louis, MO, Nov. 28-Dec. 2, 2005.

[19] Y. Shi, J. Liu, C. Jiang, C. Gao, and Y.T. Hou, "An optimal link layer model for multi-hop MIMO networks," Technical Report, the Bradley Department of Electrical and Computer Engineering, Virginia Polytechnic Institute and State University, Blacksburg, VA, July 2010. Available at http://filebox.vt.edu/users/yshi/papers/model.pdf.

[20] Q.H. Spencer, A.L. Swindlehurst, and M. Haardt, "Zero-forcing methods for downlink spatial multiplexing in multiuser MIMO channels," IEEE Transactions on Signal Processing, vol. 52, no. 2, pp. 461-471, Feb. 2004.

[21] K. Sundaresan, R. Sivakumar, M. Ingram, and T-Y. Chang, "Medium access control in ad hoc networks with MIMO links: Optimization considerations and algorithms," IEEE Transactions on Mobile Computing, vol. 3, no. 4, pp. 350-365, Oct. 2004.

[22] D. Tse and P. Viswanath, Fundamentals of Wireless Communication, Cambridge University Press, Cambridge, UK, 2005.

[23] S. Verdu, Multiuser Detection, Cambridge, UK, Cambridge University Press, 1998. 